\title{
Strongly enhanced electron-phonon coupling in the Rashba-split state of the Bi/Ag(111) surface alloy
}

\author{
Dandan Guan,,${ }^{1,2}$ Marco Bianchi, ${ }^{1}$ Shining Bao, ${ }^{2}$ Edward Perkins, ${ }^{1}$ Fabian Meier, ${ }^{3,4}$ J. Hugo Dil,${ }^{3,4}$ \\ Jürg Osterwalder, ${ }^{3}$ and Philip Hofmann ${ }^{1}$ \\ ${ }^{1}$ Department of Physics and Astronomy, Interdisciplinary Nanoscience Center, University of Aarhus, DK-8000 Aarhus C, Denmark \\ ${ }^{2}$ Department of Physics, Zhejiang University, Hangzhou, 310027 China \\ ${ }^{3}$ Physik-Institut, Winterthurerstrasse 190, Universitat Zürich-Irchel, CH-8057 Zürich, Switzerland \\ ${ }^{4}$ Swiss Light Source, Paul Scherrer Institut, CH-5232 Villigen PSI, Switzerland
}

(Received 26 January 2011; published 27 April 2011)

\begin{abstract}
The electron-phonon coupling strength of the $(\sqrt{3} \times \sqrt{3}) R 30^{\circ} \mathrm{Bi} / \mathrm{Ag}(111)$ surface alloy is investigated by temperature-dependent angle-resolved photoemission. The electron-phonon coupling strength for the Rashbasplit surface state, as expressed by the mass-enhancement parameter $\lambda$, is found to be of the order of 0.55 with little change over the investigated binding energy range between 200 and $700 \mathrm{meV}$. This coupling is much stronger than for the clean $\operatorname{Ag}(111)$ surface state or for bulk $\mathrm{Ag}$. It is, however, not found to increase near the the top of the band, where the density of states has a singularity.
\end{abstract}

DOI: 10.1103/PhysRevB.83.155451

PACS number(s): 73.20.At, 71.70.Ej, 79.60.-i

\section{INTRODUCTION}

The issues of electron lifetimes in general and the electronphonon coupling in particular are important for many chemical and physical processes and their detailed understanding is subject to a considerable amount of theoretical and experimental work. The surfaces of the noble metals have been a particular focus of attention since their electronic states can be described well by current theory ${ }^{1,2}$ and since it is possible to prepare surfaces to such high quality that lifetime investigations with angle-resolved photoemission spectroscopy (ARPES) or scanning tunneling spectroscopy (STS) become feasible (3-8 $^{3-8}$ (for a review, see Ref. 9). For the noble metals good agreement between the experimentally determined and calculated coupling strength is obtained and the electron-phonon coupling for the electronic surface states turns out to be very similar to that of the bulk states.

In this paper we investigate the electron-phonon coupling for a modified noble metal surface, the $(\sqrt{3} \times \sqrt{3}) R 30^{\circ}$ surface alloy, which is formed when $1 / 3$ of the silver atoms on $\mathrm{Ag}(111)$ are replaced by bismuth. Our study is motivated by the particular electronic character of this and similar surface alloys. $(\sqrt{3} \times \sqrt{3}) R 30^{\circ} \mathrm{Bi} / \mathrm{Ag}(111)$ supports surfacelocalized electronic states which are strongly split by the spin-orbit interaction ${ }^{10}$ and it is thus possible to investigate the electron-phonon coupling in a situation relevant to spin transport and spintronics applications.

The dispersion of the electronic states near the Brillouin zone center can be described by a Rashba model ${ }^{11-13}$ in which the free-electron-like dispersion of the electronic states is modified such that the dispersion is given by

$$
E(\mathbf{k})=E_{0}+\frac{\hbar^{2} \mathbf{k}^{2}}{2 m^{*}} \pm \alpha|\mathbf{k}|,
$$

where $E_{0}$ is an energy offset, $m^{*}$ the effective mass, and $\alpha$ a parameter measuring the strength of the spin-orbit interaction and the potential gradient at the surface. The splitting of the bands is caused by the two possible signs for the last term. It leaves the band structure completely nondegenerate, except for $\mathbf{k}=0$, where the degeneracy is protected by time-reversal symmetry. A similar spin-orbit splitting of surface electronic states is observed for materials involving heavy atoms such as $\mathrm{Au}$ (Ref. 14) or Bi (Refs. 15-18) and alloys such as $\mathrm{Bi}_{2} \mathrm{Se}_{3}$ (Ref. 19). It is also found for two-dimensional electron gases at semiconductor surfaces or interfaces but there the splitting is too small to be observed by ARPES. ${ }^{20,21}$ The splitting is absent in many bulk materials because the potential gradient which gives rise to a finite $\alpha$ is incompatible with the bulk inversion symmetry, which is often present.

The special interest in $(\sqrt{3} \times \sqrt{3}) R 30^{\circ} \mathrm{Bi} / \mathrm{Ag}(111)$ and several similar systems involving $\mathrm{Cu}$ as a substrate ${ }^{22}$ or other heavy elements on the surface, was first caused by the strong splitting of the bands ${ }^{10}$ but it soon became apparent that a genuine novelty lies in the design freedom arising from the possibility of combining different substrates, heavy atoms, and additional adsorbates. Indeed, it was established that both the strength of the spin-splitting and the position of the Fermi level can be tuned via alloying ${ }^{23,24}$ or adsorption. ${ }^{25-27}$ This is particularly interesting since it offers the possibility, at least in principle, to change the (spin) transport properties of the surface alloy. $(\sqrt{3} \times \sqrt{3}) R 30^{\circ} \mathrm{Bi} / \mathrm{Ag}(111)$ has thus been an important model system for such concepts, even though it became clear that the electronic structure ${ }^{28}$ and spin texture ${ }^{29,30}$ can deviate from a simple Rashba model.

The dispersion, density of states (DOS), and spin texture of the Rashba-split surface state on $(\sqrt{3} \times \sqrt{3}) R 30^{\circ} \mathrm{Bi} / \mathrm{Ag}(111)$ are sketched in Fig. 1. The state has a negative effective mass such that the strongly split parabola points toward higher binding energies. The dispersion is divided into two regions: region I between the top of the band and the degeneracy point at $k=0$ and region II for higher binding energies. The color of the bands is chosen such that blue denotes the inner branch and red the outer branch of the split parabola. The size of the Rashba splitting is given by the characteristic energy $E_{R}$ and wave vector $k_{0}$ defined in the figure. The spin texture at a constant energy surface is different for regions I and II. In the former the spin rotates in the same sense on both constant energy contours, whereas it rotates in the opposite sense in region II [Figs. 1(c) and 1(d)].

A particularly interesting implication of the splitting is found in the DOS which is sketched in Fig. 1(b). While a 

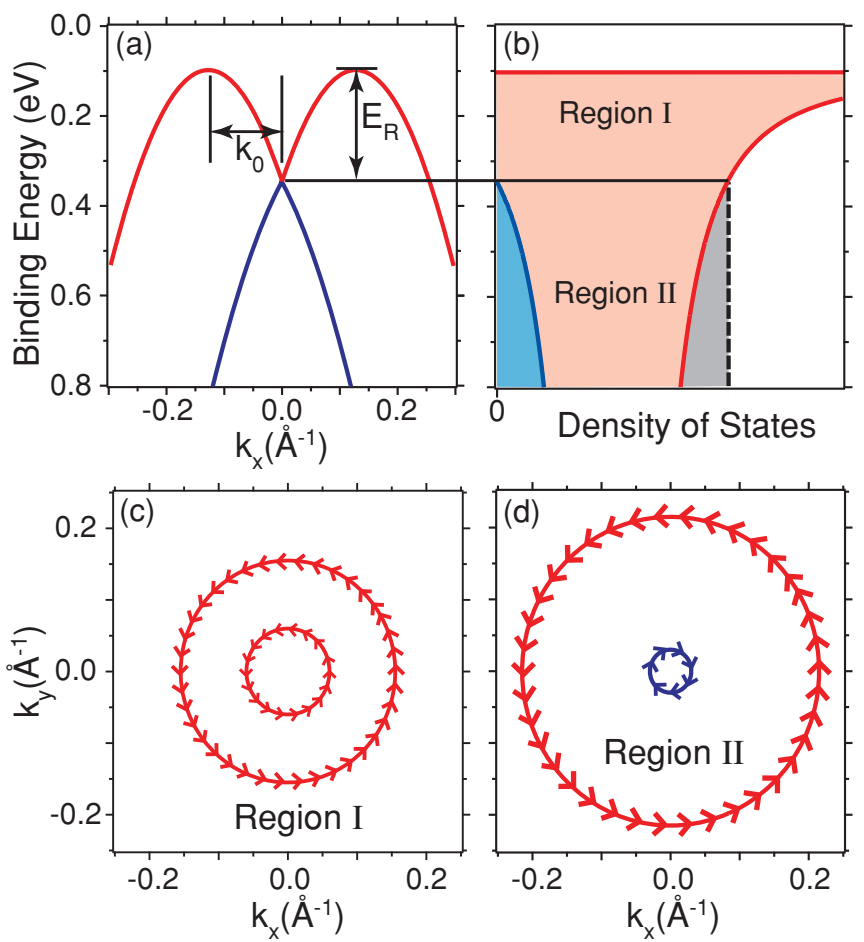

FIG. 1. (Color online) (a) Qualitative band dispersion of the Rashba-split state with the definition of the characteristic parameters describing the splitting. Red and blue are chosen for the outer and inner branch of the dispersion, respectively. (b) Corresponding DOS for the outer (red) and inner (blue) branch. The gray area represents the case without spin-orbit splitting. (c),(d) Spin texture on constant energy surfaces in the region above and below the degeneracy point.

two-dimensional free electron gas has an energy-independent DOS (shown as a gray area), this is not so for a Rashba-split state. Here the DOS is plotted separately for the inner and outer branches. The inner-branch DOS gradually goes to zero as the crossing point is approached but the outer branch shows a singularity at the top of the band.

This singularity in the DOS, which resembles a onedimensional rather than a two-dimensional situation, has been proposed to have several interesting consequences. ${ }^{31,32}$ If it is close to the Fermi energy, the singularity can be expected to have a pronounced influence on the system's properties. In particular, it can influence the strength of the effective electron-phonon coupling ${ }^{32}$ and facilitate the transition to the superconducting state. ${ }^{33}$ While the top of the band is around $100 \mathrm{meV}$ away from the Fermi energy for $(\sqrt{3} \times \sqrt{3}) R 30^{\circ}$ $\mathrm{Bi} / \mathrm{Ag}(111)$ and the state is thus irrelevant for transport properties, it has been shown that this energy can be tuned continuously by substituting $\mathrm{Bi}$ with $\mathrm{Pb}$ in the surface alloy. In fact, for the pure $(\sqrt{3} \times \sqrt{3}) R 30^{\circ} \mathrm{Pb} / \mathrm{Ag}(111)$ alloy the bottom of the band lies far above the Fermi energy. ${ }^{23}$

Systems with a tuneable Rashba splitting are also of strong interest for spintronics applications, starting from the seminal Datta Das spin field effect transistor. ${ }^{34}$ In this context it has been discussed how the combination of the Rashba effect and electron-phonon coupling can influence the localization of electrons by polaron formation. ${ }^{35-37}$ There is, however, little direct spectroscopic information on the electron-phonon coupling of Rashba systems, with the exception of $\mathrm{Au}(111)$ (Refs. 7 and 8) and the surfaces of Bi (Refs. 38-40).

Here we choose to study the electron-phonon coupling in the pure Bi surface alloy as this approach has several advantages over the mixed alloy. First of all, it is easier to achieve a reproducible preparation and the data analysis is considerably simpler if the relevant features are not too close to the Fermi energy. ${ }^{9}$ Second, the intrinsic disorder of the mixed alloy leads to enhanced defect scattering and consequently broader spectral features, ${ }^{23}$ obscuring the desired effect of the electron-phonon coupling. Finally, if the electron-phonon coupling strength is to be determined by a temperaturedependent spectroscopic study, it is not relevant if the state is close to the Fermi energy or not. This is so because only electrons within a phonon energy range can scatter into a hole by this mechanism.

\section{EXPERIMENT}

The $\operatorname{Ag}(111)$ substrate was cleaned by cycles of neon ion sputtering at $0.75 \mathrm{keV}$ for $20 \mathrm{~min}$ and annealing to $770 \mathrm{~K}$ for $10 \mathrm{~min}$. After annealing the sample was slowly cooled to room temperature at a rate of about $1 \mathrm{~K}$ per second. In the initial preparation stages, the surface cleanliness was monitored by low-energy electron diffraction (LEED). Later, ARPES from the $\operatorname{Ag}(111)$ Shockley-type surface state proved to be a much more sensitive measure of surface quality. One third of a monolayer of $\mathrm{Bi}$ was deposited by using an electron beam evaporator, while the substrate temperature was $300 \mathrm{~K}$. The deposition rate was about 3 min per monolayer. Finally, the surface was annealed to $470 \mathrm{~K}$. The coverage and quality of the surface alloy was evaluated by LEED. This is possible because a slightly higher dose of $\mathrm{Bi}$ gives rise to additional features in the diffraction pattern.

All data were taken at the SGM-3 beamline of the synchrotron radiation source ASTRID using a Specs PHOIBOS 150 hemispherical electron analyzer with a two-dimensional detector (emission angle and electron kinetic energy). The angular dispersive direction of the analyzer is perpendicular to the light's plane of incidence and the polarization vector of the light lies in the plane of incidence. The azimuthal orientation of the sample was such that the the dispersive direction of the analyzer enclosed an angle of $14^{\circ}$ with the $\bar{\Gamma} \bar{M}$ direction of the sample. All temperature-dependent data were taken in this dispersive direction, that is, as single images. Three-dimensional data sets were obtained by measuring a large set of images for different polar emission angles of the sample. The polar emission angle is defined as the angle between sample normal and the on-axis direction of the electron analyzer's lens system. All data were taken with a photon energy of $16 \mathrm{eV}$. The total energy and angular resolution were better than $10 \mathrm{meV}$ and $0.2^{\circ}$, respectively.

\section{RESULTS AND DISCUSSION}

The measured dispersion of the surface state is shown in Fig. 2 as different cuts through a three-dimensional data set. The cut in Fig. 2(a) corresponds to the schematic band structure from Fig. 1(a). It shows the characteristic double parabola. The parameters describing this band structure have 

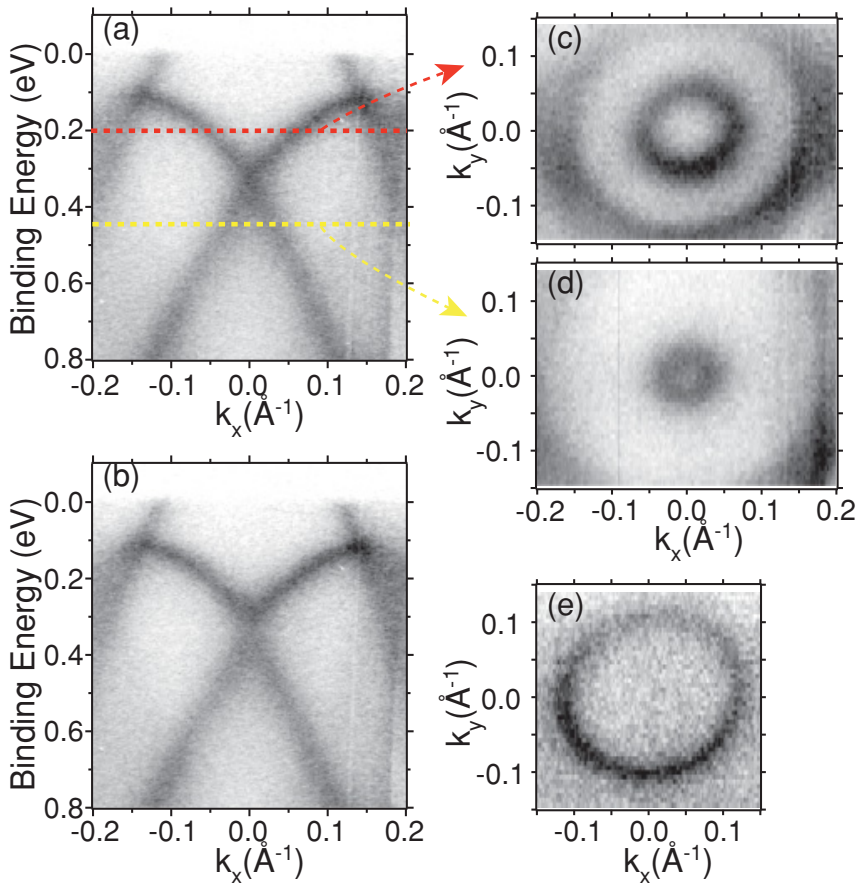

FIG. 2. (Color online) Photoemission intensity as different cuts through a three-dimensional data set. (a) Energy distribution cut through the Brillouin zone center (normal emission in the center of the image). (b) Same as (a) but with an off-normal emission angle of $0.5^{\circ}$. (c)-(e) Constant energy surfaces at binding energies of 200, 450 , and $0 \mathrm{meV}$, respectively.

been determined using a fit to the entire data set (see below) and are $E_{R}=247(69) \mathrm{meV}$ and $k_{0}=0.167(32) \AA^{-1}$, in good agreement with previous studies of the same system. ${ }^{10,23,26,29}$ Close to the band maxima additional states are observed dispersing down from the Fermi level. This is a deviation from the simple Rashba picture which we have ignored so far. First-principles calculations show that these bands have $p_{(x y)}$ character, in contrast to the Rashba state, which is $\left(s, p_{z}\right)$-like. ${ }^{28}$ Figure 2(a) shows data taken for a polar emission angle of $0^{\circ}$; that is, the center of the image corresponds to normal emission from the sample. For Fig. 2(b) the polar emission angle has been moved by $0.5^{\circ}$ away from normal emission. The resulting change in the band dispersion is small but it can easily be identified: Close to the crossing point the dispersion clearly deviates from the simple parabolic shape and the slight intensity enhancement exactly at the crossing point is lost. Such small misalignment-induced changes turn out to be detrimental to the type of data analysis reported below and have been avoided by carefully determining the normal emission direction at different temperatures.

We note that the dispersions in Figs. 2(a) and 2(b) show a considerably higher left/right symmetry in the intensity than reported in several previous experiments. ${ }^{10,29}$ The reason for this is the likely to be the experimental geometry and light polarization described above, which is completely symmetric. Residual asymmetries are mainly due to an asymmetry of the photon beam spot profile on the sample. The intensity distribution of the constant energy surfaces in Figs. 2(c)-2(e) shows the same high symmetry in the $k_{x}$ direction, as expected, but is less symmetric in the $k_{y}$ direction. This is expected because the change in $k_{y}$ is achieved by a polar rotation of the sample and thus the angle between emission direction and polarization vector is different for positive and negative $k_{y}$ values.

Information about the electron-phonon coupling in the system can be obtained from data taken at different temperatures. The basic idea behind this approach is that the lifetime of the photo-hole is limited by different possible decay processes. In the absence of magnetic excitations, these are electron-defect scattering, electron-electron scattering and electron-phonon scattering. Electron-defect scattering is independent of the sample temperature, even though the number of defects can be increased at higher temperatures. ${ }^{8}$ Electron-electron scattering is temperature dependent but only in the immediate vicinity of Fermi energy. Electron-phonon scattering has the strongest temperature dependence, essentially because the number of excited phonons and the excitation probability for phonons is strongly temperature dependent above the Debye temperature $\Theta_{D}$.

For temperatures above $\Theta_{D}$, a particularly simple description of the lifetime due to electron-phonon coupling is applicable. Indeed, while formally only valid above $\Theta_{D}$, this approximation is also rather good for temperatures around $\Theta_{D}$ and it is frequently used for assessing the electron-phonon coupling from photoemission data. In the present case, the surface Debye temperature relevant for the data is rather low, not least because of the heavy Bi atoms in the alloy ${ }^{41,42}$ The imaginary part of the corresponding self-energy $\Sigma^{\prime \prime}$, which is equivalent to half of the inverse lifetime, can be written as

$$
\Sigma^{\prime \prime}(\epsilon, \mathbf{k}, T)=\pi \lambda(\epsilon, \mathbf{k}) k_{\mathrm{B}} T+\Sigma_{0}^{\prime \prime}(\epsilon, \mathbf{k}),
$$

where $\epsilon$ and $\mathbf{k}$ are the binding energy and two-dimensional wave vector of the surface state in question, respectively, and $\lambda(\epsilon, \mathbf{k})$ is a parameter measuring the electron-phonon coupling strength. $\Sigma_{0}^{\prime \prime}(\epsilon, \mathbf{k})$ is a temperature-independent offset which takes electron-defect and electron-electron scattering into account. At the Fermi energy $(\epsilon=0), \lambda$ corresponds to the electron-phonon mass enhancement parameter ${ }^{43}$ but here we use it as a spectroscopic quantity. We further assume that $\lambda$ is energy dependent but we neglect its $\mathbf{k}$ dependence.

From the known self-energy and the single-particle dispersion of the surface state $\epsilon_{0}(\mathbf{k})$, it is possible to determine the spectral function $\mathcal{A}$ as

$$
\mathcal{A}(\epsilon, \mathbf{k}, T)=\frac{\pi^{-1}\left|\Sigma^{\prime \prime}(\epsilon, T)\right|}{\left[\epsilon-\epsilon_{0}(\mathbf{k})-\Sigma^{\prime}(\epsilon, T)\right]^{2}+\Sigma^{\prime \prime}(\epsilon, T)^{2}},
$$

assuming that $\Sigma$ is independent of $\mathbf{k}$. The real part of the self-energy $\Sigma^{\prime}$ is obtained from the imaginary part by a Kramers-Kronig transformation. It is then assumed that ARPES essentially measures the spectral function from which the temperature-dependent self-energy, and thus the electronphonon coupling strength, can be extracted.

To determine the temperature dependence of the spectral features, data similar to Fig. 2(a) were taken at seven different temperatures between $50 \mathrm{~K}$ and $350 \mathrm{~K}$. As evident from the comparison between Figs. 2(a) and 2(b), it is crucial for a quantitative analysis to use the correct angle for normal emission. Therefore, a polar angle scan was performed at several temperatures before taking the actual data. Figures 3(a) 

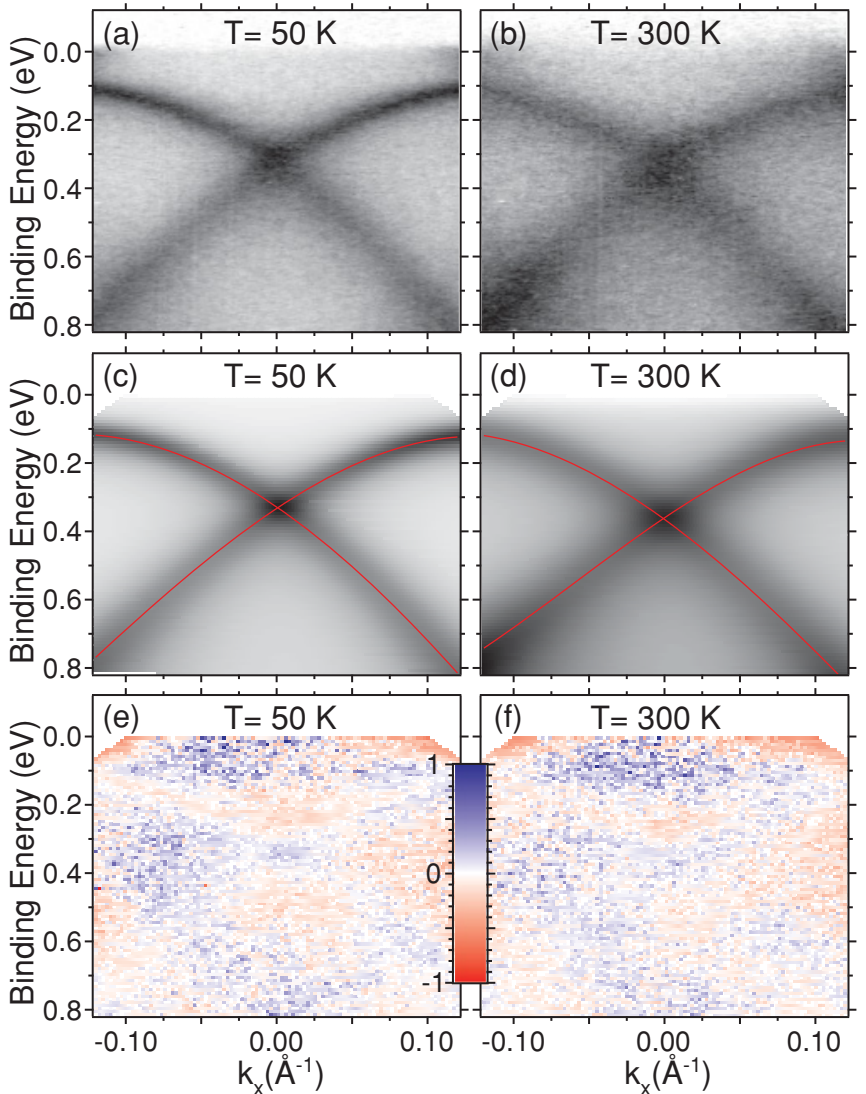

FIG. 3. (Color online) (a),(b) Photoemission spectra of the Rashba-split state, at $T=50 \mathrm{~K}$ and $T=300 \mathrm{~K}$. (b),(c) Fit to the data using a simulated spectral function (see text for details). The red lines represent the single-particle dispersion $\epsilon_{0}(\mathbf{k})$ used in the fit. (c),(d) Normalized residual between fit and data.

and 3(b) show the Rashba-split state at $50 \mathrm{~K}$ and $300 \mathrm{~K}$, respectively. It is evident that the electron-phonon coupling leads to a very significant broadening over this temperature range.

We are now interested in obtaining the temperaturedependent self-energy at different binding energies, as this can then give information about the electron-phonon coupling strength through (2). A well-established way of analyzing this type of temperature-dependent data is to consider cuts through the spectral function, either at constant emission angle or at constant binding energy. ${ }^{9}$ For the present situation such an approach cannot be used, especially not in the vicinity of the degeneracy point at the Brillouin zone center or near the top of the band. We therefore use an alternative method described by Nechaev et al. in which the entire two-dimensional data set is analyzed without performing any cuts. ${ }^{44}$

For this type of analysis, one assumes a single-particle dispersion $\epsilon_{0}(\mathbf{k})$ and a self-energy $\Sigma$ to calculate the spectral function according to (3). This spectral function is multiplied with a Fermi-Dirac distribution, broadened by the known energy and angular (or $k$ ) resolution and compared to the data via a $\chi^{2}$ calculation. Then the parameters describing the dispersion and the self-energy are refined until a satisfactory agreement is achieved.
In our case, the spectral function contains two branches, one for each Rashba-shifted parabola. $\epsilon_{0}(\mathbf{k})$ for each branch is described by a polynomial of third order. It is important to choose an order higher than two, such that small deviations from the parabolic shape can be accounted for. The energydependent intensity of each branch is also described by a third-order polynomial. According to (3) one could expect the intensity to be determined by $\Sigma^{\prime \prime}(\epsilon, T)$ but for fitting the data over an extended range of kinetic energies it is important to account for changes in the photoemission matrix elements and the intensity needs to be fitted independently of the self-energy. Finally, a model for $\Sigma^{\prime \prime}$ is needed. Any desired functional form can be used but we will see that it is sufficient to assume a linear or quadratic behavior. With the addition of a constant background, the total number of adjustable parameters in the model is thus 19 (20) for a linear (quadratic) self-energy. Despite the large number of parameters, the two-dimensional fit is stable due to the very high number of data points.

Figures 3(c)-3(f) show the fit to the data as well as the normalized residual between fit and data (the difference between model intensity and data divided by the data). The single-particle dispersion $\epsilon_{0}(\mathbf{k})$ is shown together with the fit and it is used to obtain the parameters describing the Rashba splitting, $E_{R}$ and $k_{0}$. For the comparison between data and fit, the left and right edges of the images close to the Fermi energy have been excluded because of the $p_{(x y)}$ states in these regions. A comparison of the band position and width between data and model is favorable but the residuals are not completely free of structure. This is partly ascribed to remaining small misalignments and partly to the simplicity of the model which, for example, uses a constant rather than an energy- and $k$-dependent background.

The resulting $\Sigma^{\prime \prime}$ for three temperatures is given in Fig. 4(a), both for a linear and a quadratic model The difference between these models is so small that it is hard to distinguish them on the scale of the figure. Noticeable differences exist only at the highest temperatures. In all cases $\Sigma^{\prime \prime}$ is strongly energy-dependent and decreases toward $E_{F}$. This is the expected behavior. States with higher binding energies have a shorter lifetime (larger $\Sigma^{\prime \prime}$ ) because of the electron-electron interaction, as the phase space for such scattering events increases with the binding energy. It is also apparent that $\Sigma^{\prime \prime}$ increases for higher temperatures, as expected from the electron-phonon interaction.

Figure 4(b) also shows the quadratic version of $\Sigma^{\prime \prime}$, but now plotted for a fixed binding energy and as a function of temperature. The data points correspond to the temperatures at which measurements have been taken. From the low surface Debye temperature, we expect that the data are described by (2) and the points at each temperature can indeed be fitted well by a line (solid curve). The slope of this line directly yields the electron-phonon coupling parameter $\lambda(\epsilon)$ for the binding energy $\epsilon$. The corresponding values are also given in Fig. 4(b) and plotted in Fig. 4(c).

We thus find that $\lambda$ for the $(\sqrt{3} \times \sqrt{3}) R 30^{\circ} \mathrm{Bi} / \mathrm{Ag}(111)$ surface alloy is relatively constant $(\approx 0.55)$ over the entire energy range we have investigated. It is far higher than for the surface state on the clean $\operatorname{Ag}(111)$ surface $[\lambda=0.12$ (Refs. 6 and 1)] or for bulk silver $[\lambda=0.10$ (Ref. 43)]. Without detailed theoretical model calculations, it is not possible to establish the 

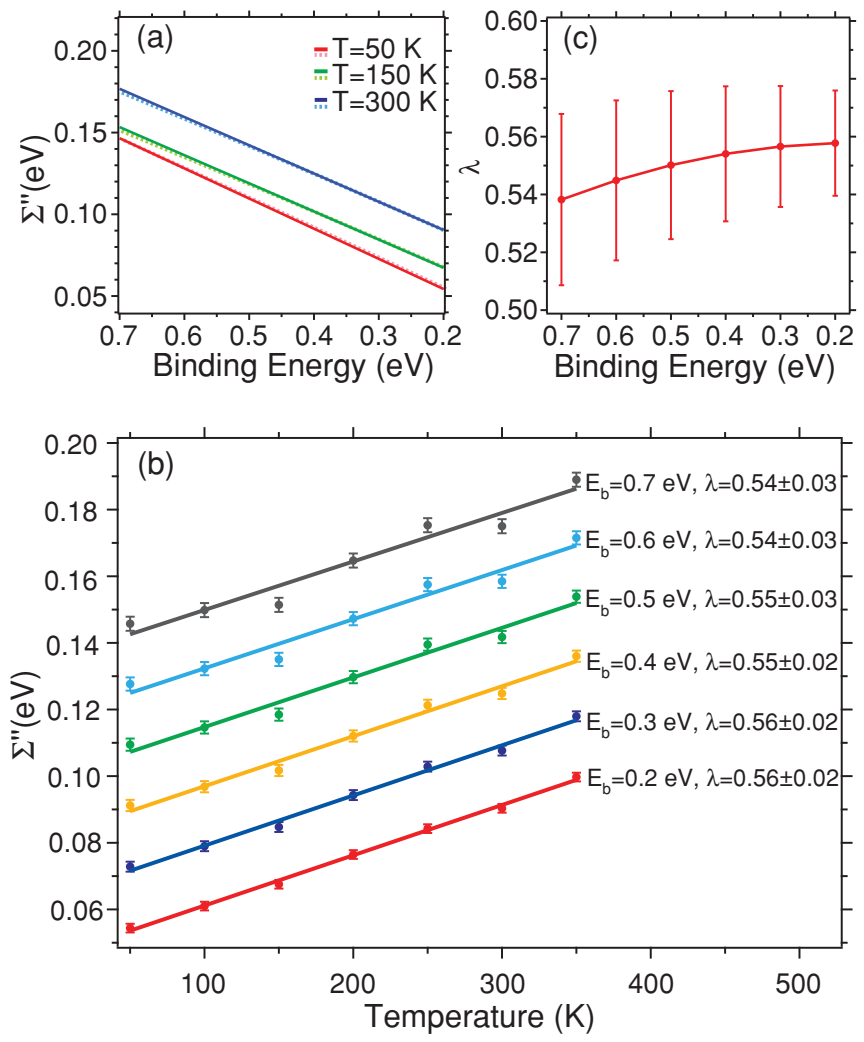

FIG. 4. (Color online) (a) Imaginary part of the self-energy $\Sigma^{\prime \prime}$, resulting from the two-dimensional fit to the data reported in Fig. 3. Solid lines represent a linear model for $\Sigma^{\prime \prime}$, dashed lines a quadratic model. The differences between the two models are very small and so the lines are difficult to distinguish. (b) Results for the parabolic model shown as a function of temperature at selected binding energies with a linear fit and the resulting values of the electron-phonon coupling constant $\lambda$. (c) $\lambda$ values plotted as function of binding energy. The uncertainties are estimated at $+5 \%$ of the minimum $\chi^{2}$ value.

origin of this enhancement as the alloy formation is severely changing both the electronic and vibrational structure close to the surface. The incorporation of $\mathrm{Bi}$ atoms, in particular, will lead to lower energy modes in the phonon spectrum.

Another interesting aspect of the result is that $\lambda$ does not vary much with the binding energy. In particular, no significant enhancement in region I is observed which might have been expected due to the singularity in the DOS. The absence of such an enhancement can be tentatively explained by the dominance of intraband scattering events involving bulk Ag states rather than interband scattering of the Rashba system. In this context, it is worthwhile pointing out that the projected $L$ gap on the $\mathrm{Ag}(111)$ surface is quite small ${ }^{45}$ and thus the Rashba-split states are not genuine surface states but surface resonances, overlapping with the projection of the bulk states.

Finally, we address the issue of a possible polaron formation, induced by the combination of Rashba splitting and electron-phonon interaction. ${ }^{35-37}$ In a recent paper, Grimaldi provides specific predictions for this possibility, also discussing the surface alloy under investigation here (see Fig. 2 in Ref. 37). For the given value of the Rashba splitting, large polaron formation can only be expected for a relatively strong electron-phonon interaction $\lambda>0.5$ and small polaron formation only for $\lambda>0.85$. Interestingly, the strong electronphonon coupling observed here opens the possibility of polaron formation for $(\sqrt{3} \times \sqrt{3}) R 30^{\circ} \mathrm{Bi} / \mathrm{Ag}(111)$, whereas this would not be possible for a coupling strength similar to that of bulk silver. On the other hand, there is little spectroscopic evidence to support the notion of polaron formation. At least the formation of small polarons is well known to severely affect the photoemission spectral line shape. ${ }^{46}$

\section{CONCLUSIONS}

In conclusion, we have determined the energy-dependent electron-phonon coupling strength for the Rashba-split state on the $(\sqrt{3} \times \sqrt{3}) R 30^{\circ} \mathrm{Bi} / \mathrm{Ag}(111)$ surface using a data analysis approach in which a model is directly fitted to the twodimensional spectral function. The electron-phonon coupling for the system is much stronger than for the clean silver surface or the bulk material but it is only weakly energy dependent. For a detailed understanding of the mechanism leading to the coupling strength enhancement, first-principles calculations of the vibrational properties and the Eliashberg coupling function would be required. However, the fact that the coupling is so strong puts the system in the interesting regime in which both the spin-orbit interaction and the electron-phonon interaction are strong and polaron formation is a possibility. It will be very interesting to see how the electron-phonon interaction is affected by tuning the strength of the Rashba interaction.

\section{ACKNOWLEDGMENTS}

Financial support from the Danish Council for Independent Research (Natural Sciences), the Carlsberg Foundation, and the Swiss National Science Foundation is gratefully acknowledged.
${ }^{1}$ A. Eiguren, B. Hellsing, E. V. Chulkov, and P. M. Echenique, Phys. Rev. B 67, 235423 (2003).

${ }^{2}$ A. Nojima, K. Yamashita, and B. Hellsing, Appl. Surf. Sci. 254, 7938 (2008).

${ }^{3}$ B. A. McDougall, T. Balasubramanian, and E. Jensen, Phys. Rev. B 51, R13891 (1995).

${ }^{4}$ J. Kliewer, R. Berndt, E. V. Chulkov, V. M. Silkin, P. M. Echenique, and S. Crampin, Science 288, 1399 (2000).

${ }^{5}$ F. Reinert, G. Nicolay, S. Schmidt, D. Ehm, and S. Hüfner, Phys. Rev. B 63, 115415 (2001).
${ }^{6}$ A. Eiguren, B. Hellsing, F. Reinert, G. Nicolay, E. V. Chulkov, V. M. Silkin, S. Hüfner, and P. M. Echenique, Phys. Rev. Lett. 88, 066805 (2002).

${ }^{7}$ S. LaShell, B. A. McDougall, and E. Jensen, Phys. Rev. B 74, 033410 (2006).

${ }^{8}$ M. Fuglsang Jensen, T. K. Kim, S. Bengio, I. Y. Sklyadneva, A. Leonardo, S. V. Eremeev, E. V. Chulkov, and P. Hofmann, Phys. Rev. B 75, 153404 (2007).

${ }^{9}$ P. Hofmann, I. Y. Sklyadneva, E. D. L. Rienks, and E. V. Chulkov, New J. Phys. 11, 125005 (2009). 
${ }^{10}$ C. R. Ast, J. Henk, A. Ernst, L. Moreschini, M. C. Falub, D. Pacile, P. Bruno, K. Kern, and M. Grioni, Phys. Rev. Lett. 98, 186807 (2007).

${ }^{11}$ E. I. Rashba, Sov. Phys. Solid State 2, 1109 (1960).

${ }^{12}$ Y. A. Bychkov and E. I. Rashba, J. Phys. C: Solid State Phys. 17, 6039 (1984).

${ }^{13}$ Y. A. Bychkov and E. I. Rashba, JETP Lett. 39, 78 (1984).

${ }^{14}$ S. LaShell, B. A. McDougall, and E. Jensen, Phys. Rev. Lett. 77, 3419 (1996).

${ }^{15}$ S. Agergaard, C. Søndergaard, H. Li, M. B. Nielsen, S. V. Hoffmann, Z. Li, and P. Hofmann, New J. Phys. 3, 15 (2001).

${ }^{16}$ Y. M. Koroteev, G. Bihlmayer, J. E. Gayone, E. V. Chulkov, S. Blügel, P. M. Echenique, and P. Hofmann, Phys. Rev. Lett. 93, 046403 (2004).

${ }^{17}$ P. Hofmann, J. E. Gayone, G. Bihlmayer, Y. M. Koroteev, and E. V. Chulkov, Phys. Rev. B 71, 195413 (2005).

${ }^{18}$ T. Hirahara, T. Nagao, I. Matsuda, G. Bihlmayer, E. V. Chulkov, Y. M. Koroteev, P. M. Echenique, M. Saito, and S. Hasegawa, Phys. Rev. Lett. 97, 146803 (2006).

${ }^{19}$ Y. Xia, D. Qian, D. Hsieh, L. Wray, A. Pal, H. Lin, A. Bansil, D. Grauer, Y. S. Hor, R. J. Cava and M. Z. Hasan, Nat. Phys. 5, 398 (2009).

${ }^{20}$ V. Y. Aristov, V. M. Zhilin, C. Grupp, A. Taleb Ibrahimi, H. J. Kim, P. S. Mangat, P. Soukiassian, and G. Le Lay, Appl. Surf. Sci. 166, 263 (2000).

${ }^{21}$ P. D. C. King, T. D. Veal, C. F. McConville, J. Zúñiga Pérez, V. Muñoz-Sanjosé, M. Hopkinson, E. D. L. Rienks, M. F. Jensen, and P. Hofmann, Phys. Rev. Lett. 104, 256803 (2010).

${ }^{22}$ L. Moreschini, A. Bendounan, H. Bentmann, M. Assig, K. Kern, F. Reinert, J. Henk, C. R. Ast, and M. Grioni, Phys. Rev. B 80 (2009).

${ }^{23}$ C. R. Ast, D. Pacile, L. Moreschini, M. C. Falub, M. Papagno, K. Kern, M. Grioni, J. Henk, A. Ernst, S. Ostanin, and P. Bruno, Phys. Rev. B 77, 081407 (2008).

${ }^{24}$ H. Mirhosseini, A. Ernst, S. Ostanin, and J. Henk, J. Phys. Condens. Matter 22, 385501 (2010).

${ }^{25}$ L. Moreschini, A. Bendounan, C. R. Ast, F. Reinert, M. Falub, and M. Grioni, Phys. Rev. B 77, 115407 (2008).

${ }^{26}$ H. Bentmann, F. Forster, G. Bihlmayer, E. V. Chulkov, L. Moreschini, M. Grioni, and F. Reinert, Europhys. Lett. 87, 37003 (2009).
${ }^{27}$ E. Frantzeskakis, A. Crepaldi, S. Pons, K. Kern, and M. Grioni, J. Electron Spectrosc. Relat. Phenom. 181, 88 (2010).

${ }^{28}$ G. Bihlmayer, S. Blugel, and E. V. Chulkov, Phys. Rev. B 75 , 195414 (2007).

${ }^{29}$ F. Meier, H. Dil, J. Lobo-Checa, L. Patthey, and J. Osterwalder, Phys. Rev. B 77, 165431 (2008).

${ }^{30}$ F. Meier, V. Petrov, S. Guerrero, C. Mudry, L. Patthey, J. Osterwalder, and J. H. Dil, Phys. Rev. B 79, 241408(R) (2009).

${ }^{31}$ C. R. Ast, G. Wittich, P. Wahl, R. Vogelgesang, D. Pacilé, M. C. Falub, L. Moreschini, M. Papagno, M. Grioni, and K. Kern, Phys. Rev. B 75, 201401 (2007).

${ }^{32}$ E. Cappelluti, C. Grimaldi, and F. Marsiglio, Phys. Rev. B 76, 085334 (2007).

${ }^{33}$ E. Cappelluti, C. Grimaldi, and F. Marsiglio, Phys. Rev. Lett. 98, 167002 (2007).

${ }^{34}$ S. Datta and B. Das, Appl. Phys. Lett. 56, 665 (1990).

${ }^{35}$ C. Grimaldi, Phys. Rev. B 77, 024306 (2008).

${ }^{36}$ L. Covaci and M. Berciu, Phys. Rev. Lett. 102, 186403 (2009).

${ }^{37}$ C. Grimaldi, Phys. Rev. B 81, 075306 (2010).

${ }^{38}$ J. E. Gayone, S. V. Hoffmann, Z. Li, and P. Hofmann, Phys. Rev. Lett. 91, 127601 (2003).

${ }^{39}$ J. E. Gayone, C. Kirkegaard, J. W. Wells, S. V. Hoffmann, Z. Li, and P. Hofmann, Appl. Phys. A 80, 943 (2005).

${ }^{40}$ C. Kirkegaard, T. K. Kim, and P. Hofmann, New J. Phys. 7, 99 (2005).

${ }^{41}$ I. M. McLeod, V. R. Dhanak, A. Matilainen, M. Lahti, K. Pussi, and K. H. L. Zhang, Surf. Sci. 604, 1395 (2010).

${ }^{42}$ I. Gierz, B. Stadtmueller, J. Vuorinen, M. Lindroos, F. Meier, J. H. Dil, K. Kern, and C. R. Ast, Phys. Rev. B 81, 245430 (2010).

${ }^{43}$ G. Grimvall, Phys. Kondens. Mater. 11, 279 (1970).

${ }^{44}$ I. A. Nechaev, M. F. Jensen, E. D. L. Rienks, V. M. Silkin, P. M. Echenique, E. V. Chulkov, and P. Hofmann, Phys. Rev. B 80, 113402 (2009).

${ }^{45}$ S. D. Kevan and R. H. Gaylord, Phys. Rev. B 36, 5809 (1987).

${ }^{46}$ L. Perfetti, H. Berger, A. Reginelli, L. Degiorgi, H. Höchst, J. Voit, G. Margaritondo, and M. Grioni, Phys. Rev. Lett. 87, 216404 (2001). 